\title{
GMPLS-Controlled \\ Dynamic Translucent Optical Networks
}

\author{
Nicola Sambo, Nicola Andriolli, Alessio Giorgetti, Luca Valcarenghi, Isabella Cerutti, \\ and Piero Castoldi, Scuola Superiore Sant'Anna \\ Filippo Cugini, CNIT
}

\begin{abstract}
The evolution of optical technologies has paved the way to the migration from opaque optical networks (i.e., networks in which the optical signal is electronically regenerated at each node) to transparent (i.e., all-optical) networks. Translucent optical networks (i.e., optical networks with sparse opto-electronic regeneration) enable the exploitation of the benefits of both opaque and transparent networks while providing a suitable solution for dynamic connections. Translucent optical networks with dynamic connections can be controlled by the GMPLS protocol suite. This article discusses the enhancements that the GMPLS suite requires for the control of dynamic translucent optical networks with quality of transmission guarantees. Such enhancements concern QoT-awareness and regenerator-awareness and can be achieved by collecting and disseminating the information on QoT and regenerator availability, respectively, and by efficiently leveraging such information for traffic engineering purposes. More specifically, the article proposes two distributed approaches, based on the routing protocol and the signaling protocol, for disseminating regenerator information in the GMPLS control plane. Moreover, three strategies are introduced to efficiently and dynamically designate the regeneration node(s) along the connection route. Routing and signaling approaches are compared in terms of blocking probability, setup time, and control plane load during provisioning and restoration.
\end{abstract}

ranslucent optical networks exploit the advantages of both transparent optical networks (where connections are switched in the optical domain) and opaque networks (where connections are optically terminated in each intermediate node and switched in the electrical domain) [1]. On the one hand, optical transparency offers considerable bandwidth at low cost. On the other hand, by performing opto-electronic signal regeneration at some of the intermediate nodes, it is possible to recover the signal degradation due to physical impairments. Both linear physical impairments (e.g., amplified spontaneous emission noise, chromatic dispersion, and polarization mode dispersion) and non-linear physical impairments due to intra- and inter-channel effects (e.g., self-phase modulation, four-wave mixing, cross-phase modulation, and cross-talk) contribute to the degradation of the optical signal quality. Such effects are especially critical for high data rates and limited wavelength spacing [2]. Opto-electronic regenerators are used to reamplify, reshape, and retime the optical signal (i.e., 3R regeneration) with the aim of guaranteeing the quality of transmission (QoT) required by the endto-end connections.

In translucent optical networks, a requested connection can be supported either by a transparent lightpath, that is, a single all-optical segment, or by a translucent lightpath, that is, a sequence of all-optical segments connected by nodes that opto-electronically regenerate the signal. Thus, a careful regenerator placement and an intelligent regenerator utilization are fundamental for designing and managing cost-effective translucent optical networks with QoT guarantees. Several studies focused on centralized schemes for regenerator placement and routing and wavelength assignment in translucent optical networks $[1,3,4]$ when connection requests are known in advance (i.e., static traffic scenario).

Translucent optical networks with dynamic connection requests present additional cross-layer challenges. In [5], a framework is proposed to address these challenges assuming that updated information is available at each node. A first challenge is the regenerator placement, which should be tailored to the dynamic scenario. Indeed, specific algorithms are required to account not only for the present and estimated future network traffic, but also to account for the dynamic provisioning and rerouting of network resources [6]. Other challenges are the QoT evaluation and the dissemination of QoT-related information. The work in [7] proposes routing solutions when QoT information is inaccurate or outdated, for example, due to coarse measurements of QoT parameters and reduced availability of monitoring equipment. Moreover, another main challenge is the study of strategies for regenerator discovery and selection. Such strategies must be designed while keeping in mind that network state information may be available only locally and may change frequently due to the dynamic nature of the connection requests. All these issues 
Path computation and wavelength assignment require QoT parameter information.

QoT parameter information can be distributed by

signaling protoco extensions routing protocol extensions
Path computation, wavelength assignment, and regenerator designation require regenerator availability information.

Regenerator information can be distributed by

signaling protocol extensions routing protocol extensions
A node has to be designated for regenerating a lightpath.

Regeneration node(s) can be designated by

\begin{tabular}{l|l|l}
$\begin{array}{l}\text { source } \\
\text { node }\end{array}$ & $\begin{array}{l}\text { intermediate } \\
\text { node }\end{array}$ & $\begin{array}{l}\text { destination } \\
\text { node }\end{array}$
\end{tabular}

Table 1. Cross-layer challenges in the control plane of dynamic distributed translucent optical networks.

influence the path computation and wavelength assignment. Preliminary studies $[2,7,8]$ address some of these challenges in control planes based on generalized multiprotocol label switching (GMPLS).

This article surveys several solutions for addressing such challenges in dynamic translucent optical networks, with a distributed control plane based on the GMPLS protocol suite, during both provisioning and restoration. Because the regenerator placement is assumed to have been decided during network design, the article focuses on the description of a QoT-aware control plane, able to distribute regenerator availability information and to dynamically designate the utilization of the information (Table 1). First, solutions for accounting and disseminating QoT information (e.g., physical impairment parameters) among control plane nodes are discussed. Second, several solutions based on GMPLS protocols are proposed for discovering available regenerators and disseminating the corresponding information among control plane nodes. Third, approaches for the selection and the reservation of the intermediate nodes that are designated for regeneration in a translucent lightpath are presented.

After a brief overview of the solutions to account for QoT in GMPLS-controlled networks, the article discusses the advantages and drawbacks of the two main approaches for the collection and distribution of regenerator information. The two proposed approaches are based on GMPLS routing and signaling protocols (i.e., Open Shortest Path First with Traffic Engineering Extensions [OSPF-TE] and Resource Reservation Protocol with Traffic Engineering Extensions [RSVPTE]). A routing-based approach advertises updated information about regenerator availability and capability and thus, can optimize the connection routing and the selection of the nodes designated for regeneration. A signaling-based approach collects regenerator information along the pre-computed connection route and avoids the advertisement of a large amount of information in the control plane. Depending on the information stored at the nodes, three strategies are defined for designating the node(s) that should perform regeneration along the translucent lightpath, that is, regenerator designation performed at source, intermediate, or destination nodes.

The performance of the proposed approaches and strategies are quantified by means of simulations. The comparison is performed in terms of blocking probability, setup time, and control-plane load. To evaluate the impact of slowly changing and rapidly changing information on regenerator availability, the performance is evaluated and compared in provisioning and restoration scenarios, respectively.

\section{QoT-Aware GMPLS Control Plane}

To guarantee the required QoT to a connection request, the GMPLS control plane must acquire physical-layer information for evaluating the QoT on each one of the all-optical seg- ments. This section presents and discusses two techniques for QoT evaluation and two approaches for disseminating QoTrelated information among network nodes.

\section{QoT Evaluation}

QoT can be evaluated through estimation or measurement. QoT estimation requires the collection of physical-layer information and the modeling of physical-layer performance. In the literature, several studies consider a single relevant parameter (e.g., equivalent length [9]) that accounts for the most detrimental physical impairment, or for several physical impairments, on each link. Then, physical-layer models combine the parameters of the links and nodes forming the alloptical segment. If the resulting QoT-estimate is within an acceptable range, the QoT of the all-optical segment is met.

Other studies propose more complex models based on multiple parameters for each physical impairment on a link. Because the various physical impairments influence each other, the difficulty of such models consists of defining dependable and flexible relationships that are able to effectively relate the several parameters. In [10], a number of physical impairments (i.e., amplified spontaneous emission, polarization-mode dispersion, chromatic dispersion, and selfphase modulation) are accounted by using a single parameter per impairment for each link. The complex model is based on the estimation of the optical signal-to-noise ratio (OSNR) penalty caused by each physical impairment as follows. Each physical impairment is estimated by combining the parameter of the links and nodes along the all-optical segment, and then it is converted into the OSNR penalty. From the OSNR, the bit-error rate (BER) is estimated. When the BER is within an acceptable range, the QoT requirements of the considered alloptical segment are met. The utilization of these models allows an a priori (i.e., a before connection set up) estimation of the QoT. The main drawback of QoT estimation is that it could be significantly complex and not sufficiently accurate.

The QoT measurement can overcome the QoT estimation complexity and inaccuracy by measuring the QoT on probe traffic or previously established transparent lightpaths. In [9], the BER is measured on probe traffic, before transmitting data. If the measured BER is acceptable, then data transmission can start. Such an approach has the drawback of delaying the connection set up due to the QoT measurement that must be performed.

\section{Dissemination of the QoT-Related Information}

The QoT-related information (e.g., physical-layer parameters or QoT measurements) can be collected and disseminated in the network by using the routing protocols or the signaling protocols of the GMPLS control plane.

When using a routing protocol, the QoT-related information is disseminated among network nodes by means of the OSPF-TE routing protocol. Each node is required to maintain a new database, referred to as a QoT-parameter database 
(QPD), which stores the physical parameters of the whole network.

When using a signaling protocol, QoT-related information is collected by RSVP-TE protocol messages. No QoT parameters are required to be stored at each node. Therefore, the signaling protocol is used for performing an online estimation of the physical impairments introduced by links and nodes traversed by RSVP-TE messages. Optionally, the signaling approach can be enhanced by introducing the QPD database at each or some of the nodes. When a node with QPD is traversed by a signaling message, the node fills the QPD with QoT parameters carried by this message.

By adopting the described approaches, the GMPLS control plane can assess the QoT for each connection to be established. When the QoT requirements cannot be met by using a single all-optical segment, regeneration is required. GMPLS extensions for handling regenerator information are discussed next.

\section{Regenerator-Aware GMPLS Control Plane}

The GMPLS control plane for a translucent optical network requires extensions for the dissemination of the information concerning the regenerator availability and for the reservation of selected regenerators.

\section{Dissemination of Regenerator-Related Information}

The GMPLS control plane can be extended to support regenerator availability information (i.e., the number of available regenerators at each node) by using routing protocols or signaling protocols. Regenerator-related information concerns both regenerator availability (i.e., regenerator state) at each node and regenerator capabilities (e.g., supported bit rate, modulation format, utilized encoding, and forward-error correction type) [11].

When using the routing protocol (i.e., OSPF-TE), each node disseminates the information about the state and the type of locally installed regenerators. Each node is required to store the received information in a local database, referred to as a regenerator database (RD). OSPF-TE is triggered upon each change of regenerator state (i.e., when a regenerator is reserved or released) [8]. An important benefit of this solution is that routing algorithms can jointly consider link (e.g., bandwidth) and node (e.g., regenerators) resources. However, this approach introduces some limitations stemming from the essence of the routing protocol (such as scalability, controlplane overload, and stability problems) when the regenerator information changes frequently (e.g., with dynamic traffic or during restoration). Therefore, the distributed routing approach seems to be more plausible when the variability of regenerator availability is moderate, that is, when the interarrival time between connection requests is long.

When using the signaling protocol (i.e., RSVP-TE), regenerator availability information is gathered during connection set up. Each node includes information about its available regenerators in the forwarded RSVP-TE messages. If the setup attempt fails, the regenerator availability information is reported to the source, which can temporarily store and exploit it for successive setup attempts of the current connection request. The signaling approach can be enhanced by introducing an RD at each node. In this case, each node traversed by the RSVP-TE messages stores the regeneration information carried by the RSVP-TE messages in the RD. Because regenerator information is not disseminated among network nodes, this approach does not suffer from the problems of control-plane overload, stability, and scalability. However, information stored in an RD might be outdated because it is updated only by RSVP-TE instances passing through the nodes.

\section{Regenerator Selection and Reservation}

The RSVP-TE is used in GMPLS-controlled networks for reserving link and node resources and also can be used for reserving regenerators. The regeneration node(s) can be designated by the source node (i.e., source designation), by any intermediate node (i.e., self designation), or by the destination node (i.e., destination designation).

- Source designation is performed at the source node when regenerator information is available in the RD. Thus, the source node routes the connection requests while taking into account the nodes with available regenerators. The source node is required to include the identifiers of the nodes designated for regeneration in the RSVP-TE Path message. Upon receiving the message, each node is informed about the source designation. Regenerator reservation takes place during the backward phase of RSVP-TE. For this reason, the destination node is required to make a copy of the list of designated regenerating nodes and to include it in the Resv message to be sent to the source node.

- Self designation is performed locally at each intermediate node. In order to meet QoT requirements, a node can designate itself for regeneration as follows. Upon receiving the RSVP-TE Path message, a node with available regenerators estimates the QoT of the all-optical segment terminating at itself. If the all-optical segment has an acceptable QoT, but it could not be extended for one more hop without exceeding the QoT requirements, then the intermediate node designates itself for opto-electronic regeneration. Designation is advertised by including its own node-identifier in the RSVP-TE Path message to be forwarded. In the backward phase, the destination node sends a Resv message to the source node containing the list of self-designated regeneration nodes. By receiving this message, each node knows whether it should reserve one of its own regenerators for the requested connection.

- Destination designation is performed at the destination node to meet QoT requirements. Upon receiving the RSVPTE Path message, the destination node designates regeneration nodes, based on regenerator availability information stored in the RD and carried by the Path message. Then, the destination node sends a Resv message to the source node containing the list of designated regeneration nodes. By receiving this message, each node knows whether it should reserve one of its own regenerators for the requested connection.

When an established connection is released, an RSVP-TE PathTear message is sent from the source to the destination. Each intermediate node receiving this message releases the regenerator (if any) reserved for such a connection.

\section{Performance Evaluation}

A performance evaluation of the GMPLS-controlled translucent optical network is performed on a Pan-European topology consisting of 27 nodes and 55 links. Each link is bidirectional and carries 40 wavelengths per direction. Seven nodes, placed according to [6], are equipped with a regenerator module composed of four regenerators. Connection requests are dynamically generated following a Poisson process and uniformly distributed among node pairs. Inter-arrival and holding times are exponentially distributed with an average of $1 / \lambda$ and $1 / \mu$ seconds, respectively. Therefore, the load offered to the network in working conditions is expressed in Erlang as the ratio $\lambda / \mu$. 


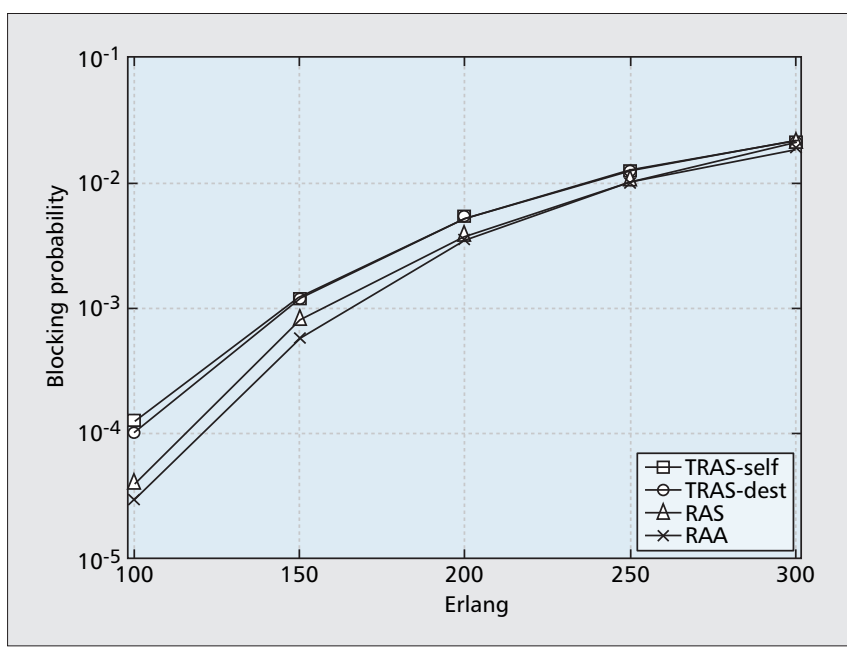

Figure 1. Provisioning blocking probability vs. network load.

The GMPLS control plane is assumed to be QoT-aware with a QPD available at each node. The QoT is estimated using an OSNR approach, as described earlier, according to the model in [10]. The wavelength availability and the QoT for the selected path are checked by consulting, respectively, the traffic engineering database (TED) and the QPD.

For each connection request, the source node $s$ selects the shortest path (in number of nodes) toward destination node $d$, among a set of candidate paths $P_{s, d}$. For each source-destination pair, $P_{s, d}$ consists of paths whose length, in number of nodes, is within the shortest path length plus one. If a transparent lightpath can be established with an acceptable QoT, RSVP-TE signaling is triggered without a GMPLS extension for regenerators. Otherwise, opto-electronic regeneration is required.

The following regenerator-aware control planes are compared:

- Control plane with regenerator availability advertisement (RAA), where regenerator availability information is disseminated by the extended OSPF-TE and stored in the RD. The path in $P_{s, d}$ with highest number of available regenerators is selected for the connection request (ties are randomly broken). Source designation of the regenerators is performed with the aim of minimizing the number of regenerators to be reserved for guaranteeing QoT.

- Control plane with regenerator availability collected by signaling (RAS), where regenerator availability information is carried by the extended RSVP-TE. The RD is filled with information received in signaling messages. The path in $P_{s, d}$ with the highest number of available regenerators is selected for the requested connection (ties are randomly broken). The source designation of the regenerators is performed at the source with the aim of minimizing the number of regenerators to be reserved for guaranteeing QoT.

- Control plane with temporary regenerator availability collected by signaling with self designation (TRAS-self), where regenerator availability information is carried by the extended RSVP-TE. The information received at the source node in signaling messages is temporarily stored until the signaling for the current connection request is terminated. In particular, at the first setup attempt, a path in $P_{s, d}$ is randomly selected, and self designation is performed. In case of setup failure, at successive setup attempts, routing and regeneration selection are performed according to the information reported to the source: the path in $P_{s, d}$ that maximizes the number of available regenerators is selected, and regeneration nodes are designated for minimizing the number of regenerators to be reserved.
- Control plane with temporary regenerator availability collected by signaling with destination designation (TRAS-dest), where regenerator availability information is carried by the extended RSVP-TE. The information received at the source node in signaling messages is temporarily stored until the signaling for the current connection request is terminated. In particular, at the first setup attempt, a path in $P_{s, d}$ is randomly selected, and destination designation is performed. In case of setup failure, at subsequent setup attempts, routing is performed according to the information reported to the source; the path in $P_{s, d}$ that maximizes the number of available regenerators is selected. At the destination, regeneration nodes are designated for minimizing the number of regenerators to be reserved.

Performance is evaluated in terms of blocking probability, setup time, and control-plane load. Results are presented in the provisioning scenario and in the restoration scenario.

\section{The Provisioning Scenario}

In the provisioning scenario, the mean connection holding time is set to $1 / \mu=10^{4} \mathrm{~s}$. The offered network load is obtained by varying $1 / \lambda$. RAS, TRAS-self, and TRAS-dest perform up to three setup attempts per connection request. RAA performs only one-set up attempt. The provisioning-blocking probability is defined as the ratio between the number of blocked connections and the number of requested connections. Blocking can be caused by unacceptable QoT (i.e., QoT blocking) due to a lack of regenerators along the selected path or by the lack of available bandwidth (i.e., the wavelength continuity constraint cannot be satisfied). The setup time is defined as the time elapsed between the connection request and the time when the connection is successfully established (i.e., the Resv message reaches the source node) and includes the propagation delay, as well as the transmission and queuing delay experienced at each traversed node. The control-plane load is measured in number of control messages (i.e., RSVP-TE and OSPF-TE messages) delivered per second. The transmission rate of the control-plane channel is $100 \mathrm{Mb} / \mathrm{s}$. Both the wavelength and the regenerator availability information are advertised upon each change of the reservation state.

Figure 1 shows the blocking probability of TRAS-self, TRAS-dest, RAS, and RAA as a function of the network load. RAA achieves the lowest blocking probability due to the prompt flooding of regenerator availability information performed by the OSPF-TE routing protocol. After three setup attempts, RAS achieves a blocking probability almost as low as RAA, while TRAS-self and TRAS-dest perform slightly worse than RAS, without requiring an RD database. The blocking probabilities of TRAS-self and TRAS-dest are comparable.

Figure 2 shows the provisioning-blocking probability of TRAS-self, TRAS-dest, and RAS after $n=1,2,3$ setup attempts as a function of the network load. RAS outperforms TRAS-self and TRAS-dest for all values of $n$ due to the information stored in the RD. However, the TRAS-self and TRAS-dest performance significantly improves for increasing $n$ due to the utilization of the regenerator availability information reported to the source node. For $n=1$ and 2, TRASdest performs slightly better than TRAS-self. Indeed, the destination has the knowledge of regenerator availability information in all the nodes along the path, while the intermediate nodes are oblivious of regenerator availability in downstream nodes. For $n=3$, TRAS-self and TRAS-dest achieve a similar blocking probability because the regenerator availability information reported at the source is almost the same after two setup attempts. RAS achieves similar per- 


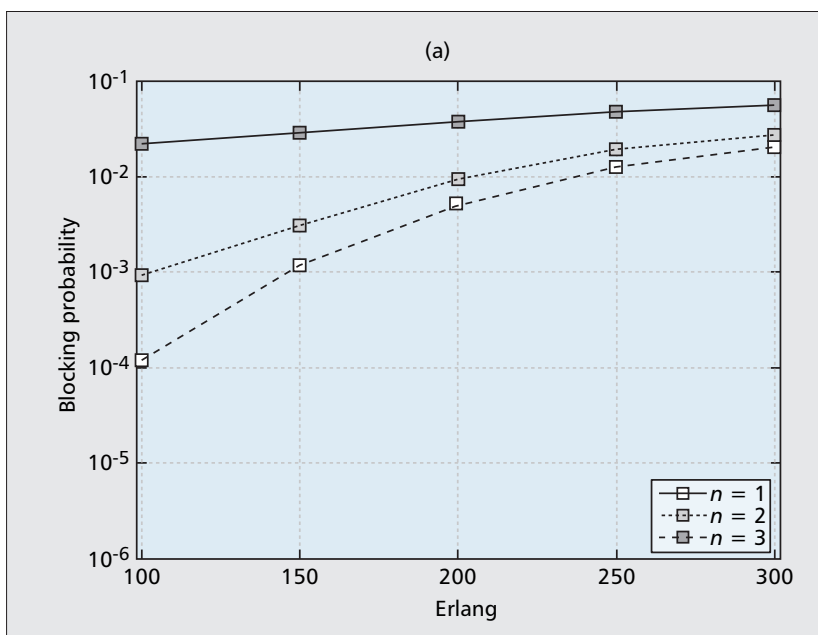

(b)

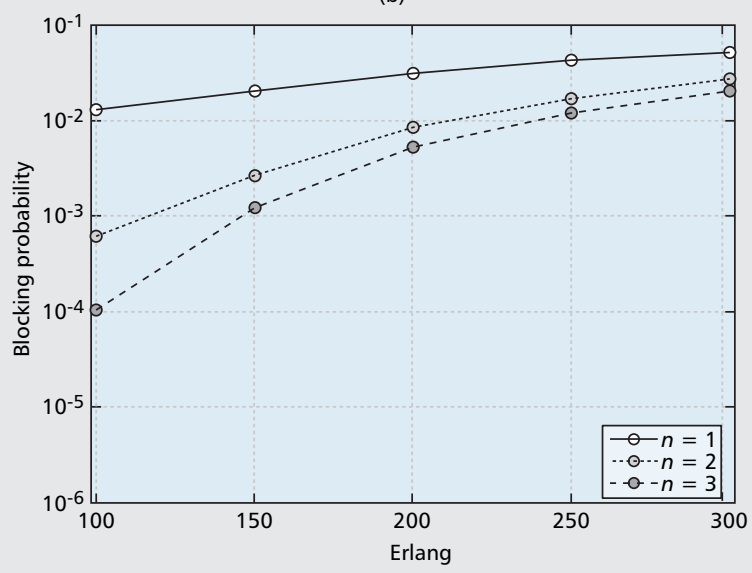

(c)

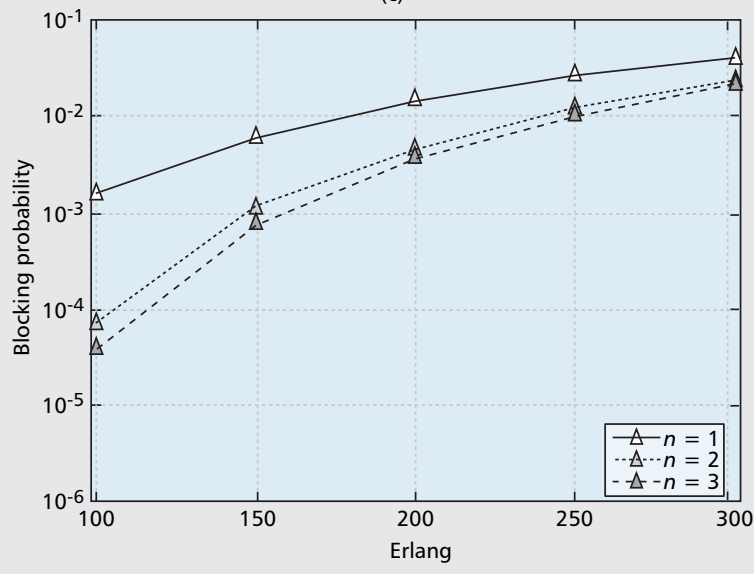

Figure 2. TRAS-self, TRAS-dest, RAS: provisioning blocking probability after $\mathrm{n}$ setup attempts vs. network load.

formance for $n=2$ and $n=3$, because it exploits the RD from the first setup attempt.

Table 2 shows the average connection setup time and the average control-plane load experienced by routing and signaling approaches when $\lambda / \mu=100,200,300$ Erlang.

The average setup time changes slightly in function of both the considered scheme and the network load. In particular, since most of the connection requests are established at the first setup attempt, the setup time is similar for all the investigated schemes. However, higher blocking at the first setup attempt (Fig. 2) implies a higher setup time. Thus, the RAA scheme, which always uses only one setup attempt, achieves the lowest setup time, whereas RAS, TRAS-dest, and TRASself experience increasing setup time.

When the load rises from 100 to 200 Erlang, the setup time increases slightly because longer paths are selected in $P_{s, d}$ to satisfy wavelength continuity constraint. However, when the load increases further to 300 Erlang, the average setup time decreases or stabilizes. At such loads, the absence of a fairness-control method causes longer connections to be blocked with higher probability. Thus, the reduction of the average setup time stems from the reduction in the average length of the established connections.

The various approaches also experience a similar controlplane load because the advertisement of regenerator availability information represents a minor contribution with respect to the advertisement of wavelength availability information.

\section{The Restoration Scenario}

In the restoration scenario, to ensure a fair comparison, the network is always provisioned by using RAS. In this scenario, different network loads are obtained by varying the holding time $1 / \mu$, while keeping the inter-arrival time $1 / \lambda$ fixed to $10^{4} \mathrm{~s}$. Single-link failures are randomly and uniformly generated.

RAA and RAS (i.e., the best performing signaling-based schemes) are utilized as path restoration schemes to restore disrupted connections. Upon link failure, the node detecting the failure (e.g., the downstream node of the failed link) sends a notification to the source node, which sends an RSVP-TE PathTear message to release resources. Then, the source node selects a backup path from the source to the destination within $P_{s, d}$ (considering the network topology without the failed link) as previously described, and RAA or RAS is performed. During restoration, RAS and RAA perform one setup attempt for restoring each connection.

For comparison purposes, a segment restoration scheme (SRS) [12], based on a signaling protocol is presented. The SRS performs restoration of the failed all-optical segment. Upon link failure, the node detecting the failure sends a notification to the source node of the failed alloptical segment (i.e., branch node). The branch node computes an alternative route to the destination node of the all-optical segment (i.e., merge node). If required, it designates the regenerator node(s) (as in the source designation) by using the information stored in the RD. The SRS has the advantage that the restoration connection can exploit the regenerators already reserved by the working connection.

The restoration-blocking probability is defined as the ratio between the number of unsuccessfully restored connections and the number of connections affected by the fault. A restoration attempt can be blocked due to unacceptable QoT (i.e., QoT blocking) and due to wavelength contention/unavailability. The restoration setup time is defined as the time between the failure and the time when the connection is successfully restored. The controlplane load is measured in number of control messages per failure.

Figure 3 shows the overall restoration-blocking probability and the QoT-blocking probability contribution experienced by RAA, RAS, and SRS. The restoration blocking increases with the offered network load due to the increase of concurrent reservation instances. RAS and RAA experience similar QoT and overall blocking probability. Even though the number of regenerators in the network is limited, the regenerator availability information disseminated with RAA does not converge in due time, thus it does not improve the blocking obtained by 


\begin{tabular}{|l|l|l|l|l|l|l|}
\multirow{2}{*}{ Control plane } & \multicolumn{4}{|c}{ Average setup time (ms) } & \multicolumn{3}{c}{ Average control plane load (packets/s) } \\
\cline { 2 - 8 } & 100 Erlang & 200 Erlang & 300 Erlang & 100 Erlang & 200 Erlang & 300 Erlang \\
\hline RAA & 13.20 & 13.10 & 12.81 & 11.55 & 16.45 & 20.75 \\
\hline RAS & 13.24 & 13.39 & 13.38 & 10.13 & 14.87 & 19.09 \\
\hline TRAS-dest & 13.48 & 13.84 & 13.85 & 10.12 & 14.83 & 19.03 \\
\hline TRAS-self & 13.78 & 14.04 & 13.98 & 10.12 & 14.83 & 19.03 \\
\hline
\end{tabular}

- Table 2. Average setup time and control plane load during provisioning.

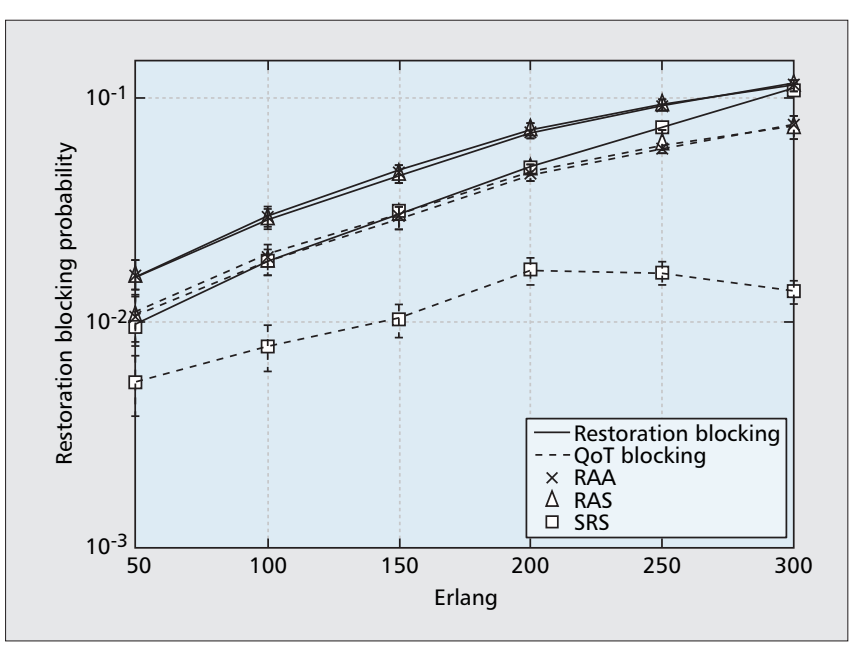

Figure 3. Restoration blocking probability vs. network load.

\begin{tabular}{|lll|}
\hline Control plane & Setup time & Control plane load \\
\hline RAA & 19.67 & 624.64 \\
\hline RAS & 19.69 & 295.92 \\
\hline SRS & 17.15 & 277.46 \\
\hline
\end{tabular}

- Table 3. Average setup time (ms) and control plane load (packets per failure) during restoration, at 200 Erlang.

RAS. On the contrary, this dissemination significantly increases the control-plane load during restoration (see RAA in Table 3). The increased control-plane load, due to regenerator availability advertisement, is evident during the restoration phase because the wavelength availability information is not advertised.

SRS strongly reduces QoT blocking and consequently, the overall restoration blocking with respect to RAA and RAS. In SRS, by utilizing regenerators reserved by the failed connections, fewer regenerators must be specifically reserved during restoration, and thus, inaccurate regenerator availability information in RD has a lower impact on the blocking. RAA and RAS achieve similar restoration setup times, whereas SRS achieves the lowest restoration setup time because signaling messages are exchanged only between branch and merge nodes. Finally, the number of control-plane messages sent during restoration is lower in RAS and SRS than in RAA because the former ones do not disseminate any regenerator availability information (Table 3).

\section{Conclusions}

This article presents the most relevant open issues concerning a GMPLS control plane supporting a translucent optical network with QoT guarantees: first, how to evaluate the physical-impairment impact on the connection QoT; second, how to collect and disseminate regenerator availability information among network nodes; and third, how to reserve and release a regenerator resource. Schemes based on both routing protocols and signaling protocols were presented to enforce GMPLS-controlled translucent optical networks.

The approaches were tested in both provisioning and restoration scenarios. A comparison indicates that the routing approach achieves better performance during provisioning but suffers from scalability issues due to the large amount of disseminated information, especially under dynamic traffic conditions, for example, during restoration. Signaling approaches avoid the scalability issues but may require multiple attempts (leading to a longer connection setup time) to achieve similar performance in terms of blocking.

Finally, segment restoration between opto-electronic regenerators overcomes the problem of inaccurate regenerator information at the node databases and guarantees a faster restoration time. This finding may call for novel provisioning and restoration approaches for dynamic translucent optical networks.

\section{Acknowledgment}

The work described in this article was performed with the support of the Building the Future Optical Network in Europe (BONE) project, a Network of Excellence funded by the European Commission through the 7th ICT-Framework Programme and by MIUR under the FIRB project, Software and Communication Platforms for High-Performance Collaborative Grid (RBIN043TKY).

\section{References}

[1] S. Gangxiang and R. Tucker, "Translucent Optical Networks: The Way Forward," IEEE Commun. Mag., vol. 45, no. 2, Feb. 2007, pp. 48-54.

[2] S. Sygletos, I. Tomkos, and J. Leuthold, "Technological Challenges on the Road toward Transparent Networking," OSA J. Optical Net., vol. 7, no. 4, Apr. 2008, pp. 321-50

[3] I. Cerutti and A. Fumagalli, "Traffic Grooming in Static Wavelength Division Multiplexing Networks," IEEE Commun. Mag., vol. 43, no. 1, Jan. 2005, pp. 101-07.

[4] G. Shen and W. D. Grover, "Segment-Based Approaches to Survivable Translucent Network Design under Various Ultra-Long-Haul System Reach Capabilities," OSA J. Optical Net., vol. 3, no. 1, Jan. 2004, pp. 1-24.

[5] X. Yang and B. Ramamurthy, "Dynamic Routing in Translucent WDM Optical Networks: The Intradomain Case," J. Lightwave Tech., vol. 23, no. 3, Mar. 2005, pp. 1713-23.

[6] X. Yang and B. Ramamurthy, "Sparse Regeneration in Translucent Wavelength-Routed Optical Networks: Architecture, Network Design and Wavelength Routing," Photonic Net. Commun., 2005.

[7] R. Martinez et al., "Challenges and Requirements for Introducing ImpairmentAwareness into the Management and Control Planes of ASON/GMPLS WDM Networks," IEEE Commun. Mag., vol. 44, no. 12, Dec. 2006, pp. 76-85. 
[8] N. Sambo et al., "Distributing Shared Regenerator Information in GMPLS Controlled Translucent Networks," IEEE Commun. Letters, vol. 12, no. 6, June 2008, pp. 462-64.

[9] N. Sambo et al., "Probe-Based Schemes to Guarantee Lightpath Quality of Transmission (QoT) in Transparent Optical Networks," Proc. Euro. Cont Optical Commun. (ECOC 2008), Sept. 2008.

[10] F. Cugini et al., "Enhancing GMPLS Signaling Protocol for Encompassing Quality of Transmission (QoT) in All-Optical Networks," J. Lightwave Tech., vol. 26, no. 19, Oct. 2008, pp. 3318-28.

[11] G. Bernstein, Y. Lee, and W. Imajuku, "Framework for GMPLS and PCE Control of Wavelength Switched Optical Networks," Internet draft, Oct 2007; draft-bernstein-ccampwavelength-switched-02.txt

[12] N. Sambo et al., "Segment Restoration Scheme with QoT-Guarantees in GMPLS-Controlled Translucent Networks," Proc. Euro. Conf. Optical Commun. (ECOC 2008), Sept. 2008.

\section{Biographies}

NICOLA SAMBO [S] received a Laurea degree in telecommunication engineering from the University of Pisa, Italy, in 2004. He is currently pursuing a Ph.D. degree at Scuola Superiore Sant'Anna, Pisa, Italy, in the Centre of Excellence for Information and Communication Engineering (CEIIC). His research interests include WDM network planning and modeling, QoS and fault tolerance in optical networks, and the GMPLS protocol suite for optical networks. In 2007 he was a visiting student at France Télécom, Lannion.

NICOLA ANDRIOLLI received a Laurea degree in telecommunications engineering from the University of Pisa in 2002, and Diploma and Ph.D. degrees from Scuola Superiore Sant'Anna in 2003 and 2006, respectively. Currently, he is an assistant professor at Scuola Superiore Sant'Anna. His research interests include the design and analysis of circuit- and packet-switched optical networks and nodes. He was a visiting student at BUTE, Budapest; DTU Fotonik, Copenhagen; and NICT, Tokyo

ALESSIO GIORGETTI (alessio.giorgetti@sssup.it) received a Laurea degree in telecommunications engineering from the University of Pisa in 2002 and a Ph.D. degree from Scuola Superiore Sant'Anna in 2006. He is currently a post-doctor- al fellow with Scuola Superiore Sant'Anna. His research interests include wavelength-division multiplexing network design, fault tolerance in optical networks, and GMPLS control plane for wavelength-routed optical networks. He was a vis iting scholar at the Centre for Photonic Systems, University of Cambridge, United Kingdom.

ISABELLA CERUTTI [M'03] holds a Laurea degree in electrical engineering from the Politecnico di Torino, Italy (1998), and a Ph.D. in electrical engineering from the University of Texas at Dallas (UTD, 2002). From 2002 to 2006 she was a post-doctoral research associate at UTD and then at Scuola Superiore Sant'Anna. Since 2007 she has been an assistant professor at SSSUP. She has co-authored more than 50 publications in international journals and conference proceedings.

FILIPPO CUGINI received an M.S. degree in telecommunications engineering from the University of Parma, Italy. Since 2001 he has been with the National Laboratory of Photonic Networks, Consorzio Nazionale Interuniversitario per le Telecomunicazioni (CNIT), Pisa, Italy. His main research interests include MPLS, GMPLS, and PCE protocols and architectures, survivability in IP-over-WDM networks, and traffic engineering in grid networking.

LUCA VALCARENGHI [S'99, $\mathrm{M}^{\prime} 01$ ] holds a Laurea degree in electronics engineering (1997) from the Politecnico di Torino, Italy, and an M.S.E.E. (1999) and a Ph.D. in electrical engineering-telecommunications (2001), both from UTD. Since September 2002 he has been an assistant professor at the Scuola Superiore Sant'Anna. His main research interests are optical network design, analysis, optimization, and reliability; QoS in network infrastructures for grid computing; and fixed and mobile network integration.

PIERO CASTOLDI [S'93, M'07] received a Laurea degree in electrical engineering from the University of Bologna, Italy, in 1991, and a Ph.D. degree from the University of Parma in 1996. In 1996 and 1997 and the summers of 1999 and 2000, he was a post-doctoral fellow in the Department of Electrical Engineering, Princeton University, New Jersey. Since March 2001 he has been an associate professor in telecommunications at the Scuola Superiore Sant'Anna, where he is currently responsible for the networks area. From December 1997 to February 2001 he was an assistant professor with the Faculty of Engineering, University of Parma.

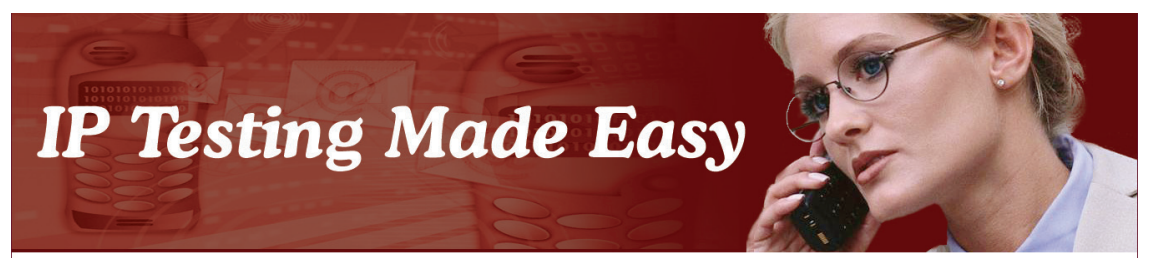

PacketCheck ${ }^{\mathrm{TM}}$

\section{- Generate or Receive Full Duplex IP, UDP, Ethernet Traffic}

- Bit-Error-Rate Testing on Layer 2 / 3 / 4 (User defined pattern, Sequence number)

- Monitor Performace Statistics (Throughput, Packet loss, Out of order packets)

- Control Bandwidth in Real Time

- Frame Lengths Supported 64 bytes to 1518 bytes

- Detailed Test Reports

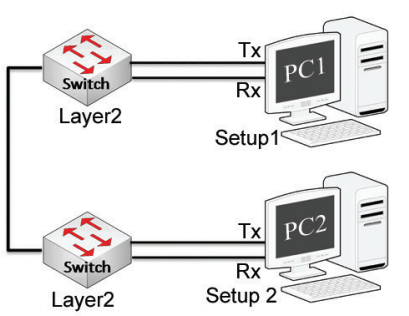

Ethernet BER Test Setup at Layer 2

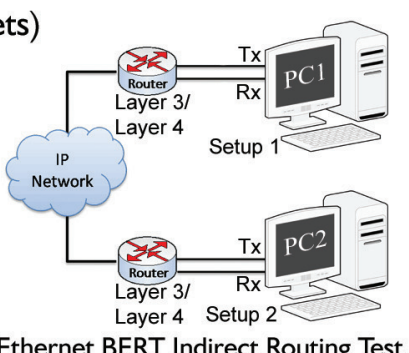

Ethernet BERT Indirect Routing Test Setup at Layer 3/ Layer 4

\section{GL Communications Inc.}

301-670-4784*info@gl.com *www.gl.com 